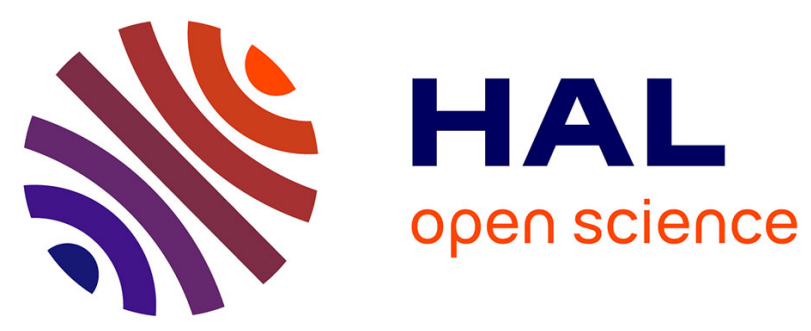

\title{
Physiological pathway for low density lipoproteins across the blood-brain barrier: transcytosis through brain capillary endothelial cells in vitro
}

Pietra Candela, Fabien Gosselet, Florence Miller, Valérie Buée-Scherrer, Gérard Torpier, Roméo Cecchelli, Laurence Tilloy-Fenart

\section{To cite this version:}

Pietra Candela, Fabien Gosselet, Florence Miller, Valérie Buée-Scherrer, Gérard Torpier, et al.. Physiological pathway for low density lipoproteins across the blood-brain barrier: transcytosis through brain capillary endothelial cells in vitro. Endothelium, 2008, p. 254-264. hal-00543686

\section{HAL Id: hal-00543686 \\ https://hal-univ-artois.archives-ouvertes.fr/hal-00543686}

Submitted on 19 Jul 2021

HAL is a multi-disciplinary open access archive for the deposit and dissemination of scientific research documents, whether they are published or not. The documents may come from teaching and research institutions in France or abroad, or from public or private research centers.
L'archive ouverte pluridisciplinaire HAL, est destinée au dépôt et à la diffusion de documents scientifiques de niveau recherche, publiés ou non, émanant des établissements d'enseignement et de recherche français ou étrangers, des laboratoires publics ou privés.

\section{(ㄷ)(1)}

Distributed under a Creative Commons Attribution| 4.0 International License 


\title{
Physiological Pathway for Low-Density Lipoproteins across the Blood-Brain Barrier: Transcytosis through Brain Capillary Endothelial Cells In Vitro
}

\author{
Pietra Candela, Fabien Gosselet, Florence Miller, and Valérie Buee-Scherrer \\ Laboratoire de Physiopathologie de la BHE, Faculté des Sciences Jean Perrin, rue Jean Souvraz, \\ Lens Cedex, France

\section{Gérard Torpier} \\ U 545-INSERM, Département d'Athérosclérose, Institut Pasteur de Lille, Lille, France \\ Roméo Cecchelli and Laurence Fenart \\ Laboratoire de Physiopathologie de la BHE, Faculté des Sciences Jean Perrin, rue Jean Souvraz, \\ Lens Cedex, France
}

\begin{abstract}
Although an immense knowledge has accumulated concerning regulation of cholesterol homeostasis in the body, this does not include the brain, where details are just emerging. Using an in vitro blood-brain barrier model, the authors have demonstrated that low-density lipoprotein (LDL) underwent transcytosis through the endothelial cells (ECs) by a receptor-mediated process, bypassing the lysosomal compartment. Moreover, caveolae might be involved in these blood-borne molecule transports from the blood to the brain. Although several ligands are known to be internalized through cell surface caveolae, the subsequent intracellular pathways have remained elusive. By cell fractionation experiment and Western blot, the authors have demonstrated that the LDL receptor is located in the caveolae membrane fraction. Then, LDLs internalized were detected by electron microscopy in multivesicular bodies. The authors identified in brain capillary ECs a novel endosomal compartment, mildly acidic, positive for marker Lamp1 but devoid of any degradative capability. From the point of view of $\mathrm{pH}$, cellular location, and caveolae-derived formation, the multivesicular organelles described here can be related to the caveosome structure. These results could provide clues to physiological functions of caveolae-caveosome transcellular pathway in brain capillary ECs and may help in the rational design of more effective therapeutic drugs to the brain.
\end{abstract}

Received 16 May 2008; accepted 27 July 2008. 2008)

This work is dedicated to the memory of Gérard Torpier (1943-

This work is supported by grants from the Ministry of Research of France (fellowship to P. Candela). Florence Miller was supported by Région Nord-Pas de Calais and Cellial Technologies. The authors acknowledge equipment provided by the "Service Commun d'imagerie cellulaire de L'Université Lille 2 mis en place par I'IMPRT (IFR 114)." The authors thank Mrs. A. M. Lenfant for her technical assistance.

Address correspondence to Pr Laurence Fenart, Laboratoire de physiopathologie de la BHE, Faculté Jean Perrin, rue jean Souvraz, SP 18,62307 Lens Cedex, France. E-mail: laurence.fenart@univ-artois.fr
Keywords Blood-Brain Barrier, Caveolae, Endocytosis, Endothelial Cells, Low-Density Lipoproteins, Transcytosis

The brain is the most cholesterol-rich organ in the body and contains about $25 \%$ of the total amount, most of it present in myelin. Unlike peripheral endothelium, brain capillary endothelial cells (ECs), constituting the blood-brain barrier (BBB), are characterized by the presence of highly resistant, tight intercellular junctions, minimal pinocytic activity, and absence of fenestration (Betz and Goldstein 1978). These characteristics endow the brain capillary ECs with the ability to restrict the passage of most polar blood-borne molecules from the cerebrovascular circulation to the brain, including cholesterol. Indeed, cholesterol is essential in the cellular metabolism of all mammalian tissues, including brain (Morris et al. 1992). Even if it was commonly allowed that de novo synthesis is responsible for practically all cholesterol present in the brain, some results argued also in favor of existence of some exchanges between plasma and brain cholesterol. Indeed, cholesterol changes in the extracerebral cholesterol levels are readily sensed by the low-density lipoprotein (LDL) receptor in the brain, causing appropriate modifications in its activity (Malavolti et al. 1991). Owing to the fact that the neurological abnormalities that result from the inadequate absorption of diatery vitamin E can be improved by the oral administration of pharmacological doses of vitamin E, Traber and Kayden (1984) have suggested that LDL functions as a transport system for tocopherol to the brain. Furthermore, the trace amounts of apolipoprotein B in cerebrospinal fluid confirm that, at most, small amounts of apolipoprotein B normally pass through the BBB. 
LDL receptors are expressed on brain microvascular endothelia in humans but not on capillary beds in peripheral tissues(Méresse et al. 1989). Unlike large vessels in vivo(Vasile et al. 1983) and in culture(Kenagy et al. 1984), confluent brain capillary ECs express an LDL receptor (Méresse et al. 1991). Moreover, its expression is enhanced by cocultivation of ECs with astrocytes (Lucarelli et al. 2002; Dehouck et al. 1994). The discovery of these unexpectedly expressed receptors on the luminal side of the brain capillary ECs implied that cholesterol might cross the BBB, although most aspects of this pathway were still unclear.

Using an in vitro BBB model, which closely mimics the in vivo situation(Lundquist et al. 2002; Cecchelli et al. 2007), we have found evidence for the selective transport of LDLs across the brain capillary EC monolayers. This transport was completely inhibited at low temperature, indicating that LDLs are directed to the abluminal compartment by a transcellular route. Moreover, using the conventional antireceptor antibodies, a complete inhibition of the LDL uptake was observed(Dehouck et al. 1997). Taken together, these results led us to conclude that LDLs are transcytosed through the BBB by way of a specific receptor-mediated pathway. The recent results from Spencer and Verma (2007) demonstrating a specific targeted brain delivery by using apolipoprotein $\mathrm{B}$ (ApoB)-fused proteins are in total accordance with our results.

With respect to the delivery and transport of pharmaceuticals, better characterization of this transcytotic pathway should lead to advances in the development of new transcellular delivery systems through the BBB. Moreover, the importance of understanding cholesterol traffic through brain capillary ECs is underscored by the accumulating and convincing evidence that an association exists between plasma cholesterol and development of neurodegenerative disorders such as Alzheimer and Niemann-Pick diseases (Howland et al. 1998; Refolo et al. 2000; Shie et al. 2002). Recently, Cao et al (2006) suggest that the LDL receptor plays a role in the development of Alzheimertype learning impairment and amyloidosis and can be a novel therapeutic target for Alzheimer disease. All these new results clearly emphasize the importance of understanding the different step of this transcytotic pathway and may help in the rational design of more effective therapeutic drugs.

In the present study, confocal microscopy and immunogold eletronmicroscopy were used to investigate the caveolaedependent transcytotic pathway of LDL movement across the BBB. We characterized a novel endosomal compartment involved in the LDL transcytosis: caveolin $1-$ and $\beta$-COPpositive, mildly acidic, and devoided of any degradative capabilities.

\section{METHODS}

\section{Antibodies}

Antibodies were as follows: rabbit polyclonal anti-caveolin 1 antibody (C13630; Transduction Laboratories, Lexington, KY); chicken polyclonal antibody to human LDL-receptor (Progen, Heidelberg, The Netherlands); mouse polyclonal anti-paxillin antibody was a gift from R. Béliveau (Montréal, Canada); rabbit anti-Lamp 1 was a gift from S. Méresse (Marseille, France); polyclonal goat anti-ApoB (developed in the laboratory); monoclonal anti- $\beta$-COP antibody (Sigma-Aldrich, Saint Quentin Fallavier, France); monoclonal anti-human golgin97 antibody (Molecular Probes, Eugene, OR); CY5 conjugated goat antirabbit immunoglobulin G (IgG) (Molecular Probes); Alexa 488conjugated goat anti-rabbit IgG, Alexa 488-conjugated rabbit anti-mouse IgG, Alexa 568-conjugated donkey anti-goat $\operatorname{IgG}$ (Molecular Probes); peroxidase-conjugated rabbit anti-chicken IgY (Jackson Immunoresearch laboratories, West Grove, PA); peroxidase-conjugated goat anti-mouse IgG (Sanofi- Diagnostics Pasteur, Gif sur Yvette, France); DiI LDL and DiI acetylated LDL (Molecular Probes, Eugene, OR).

\section{Cell Culture}

The experimental procedure was as follows: Cultures of astrocytes were prepared from newborn rats as described by Booher and Sensenbrenner (1972). Astrocytes were plated on six well dishes (Nunclon; Nunc A/S, Roskilde, Denmark) at a concentration of $3 \times 10^{4} \mathrm{cells} / \mathrm{ml}$ in $2 \mathrm{ml}$ of Dulbeco's modified Eagle's medium (DMEM) supplemented with $10 \%$ fetal calf serum (Gibco Life Technologies) and the medium was changed twice a week.

After 3 weeks, cultures of astrocytes were stabilized and coated filters were set in 6-well dishes containing astrocytes. ECs, isolated from bovine brain and characterized as described by Méresse et al. (1989), were plated at a concentration of 4 $\times 10^{5}$ cells $/ \mathrm{ml}$ on the upper side of the coated filters (Bornstein 1958) in $1.5 \mathrm{ml}$ of medium. The medium shared by both cell types was DMEM supplemented with $10 \%(v / v)$ heatinactivated calf serum, $10 \%(v / v)$ horse heat-inactivated serum (Gibco Life Technologies), $2 \mathrm{mM}$ glutamine, $50 \mu \mathrm{g} / \mathrm{ml}$ gentamycin, and basic fibroblast growth factor (bFGF; $1 \mathrm{ng} / \mathrm{ml}$, added every other day).

This medium was changed every other day. Under these conditions, ECs form a confluent monolayer in 7 days. Experiments were performed 5 days after confluence. This arrangement readily permits the use of different cell types, which were separated easily after coculture by removing the insert.

For all experiments that required ECs, the results presented are representative of three series of independent experiments. The immunostaining of tight junction proteins is routinely performed in the laboratory.

\section{Purification of Caveolae and Immunoblots}

Plasma membrane fractions from up-regulated (Dehouck et al. 1994) or non-up-regulated bovine brain capillary ECs cocultivated with astrocytes during 12 days, isolated as described by Smart et al. (1995), served as the starting material. After sonication, caveolin-containing microdomains were separated 
from residual plasmalemmal domains using two successive Optiprep gradients (Smart et al. 1995). Plasma membrane fractions and caveolar membrane fraction were concentrated by trichloroacetic acid precipitation and washed in acetone. Pellets were suspended in Laemmli sample buffer and heated at $95^{\circ} \mathrm{C}$ for $3 \mathrm{~min}$ before being loaded onto gels. Proteins were separated in a $4-\%$ to $12 \%$ gel (NuPage TM $4-12 \%$ Bis Tris gel; VWR). The separated proteins were then transferred to nitrocellulose membrane. The membrane was blocked in phosphate-buffered saline (PBS) that contained $10 \%$ dry milk for $1 \mathrm{~h} 30 \mathrm{~min}$ at room temperature. It was then incubated for $2 \mathrm{~h}$ at room temperature in primary antibodies (either with chicken polyclonal antibody against human LDL receptor or with mouse polyclonal antibody against paxillin) diluted in PBS that contained 3\% dry milk. The membrane was washed five times with PBS. The secondary antibodies (horseradish peroxydase-conjugated antibodies directed against chicken or mouse IgGs) were diluted at 1:5000 in PBS containing 3\% dry milk and applied overnight at $4^{\circ} \mathrm{C}$. The membrane was then washed and the bands were visualized using enhanced chemiluminescence detection (Amersham Laboratories, UK).

\section{Preparation of LDL and Acetylated LDL}

LDL was isolated from human plasma by sequential ultracentrifugation at densities of 1.03 to 1.053 . The densities were adjusted using solid $\mathrm{KBr}$. The $\mathrm{LDL}$ was extensively dialyzed at $4^{\circ} \mathrm{C}$ against $0.15 \mathrm{M} \mathrm{NaCl}$ and against DMEM. Acetylated LDL was prepared by treating LDL with acetic anhydride (Basu et al. 1976) and extensively dialyzed at $4^{\circ} \mathrm{C}$ against DMEM.

\section{Fluorescence Microscopy}

ECs grown on porous filters were fixed at room temperature for $20 \mathrm{~min}$ with $4 \%$ paraformaldehyde in a fibrous components-stabilizing buffer $(60 \mathrm{mM}$ piperazine$N, N^{\prime}$-bis(2-ethanesulfonic acid) [PIPES], $25 \mathrm{mM} \mathrm{N}$-2hydroxyethylpiperazine- $N^{\prime}$-2-ethanesulfonic acid (HEPES), 10 mM EGTA, $2 \mathrm{mM} \mathrm{MgCl}_{2}$, and $140 \mathrm{mM} \mathrm{NaCl}$, pH 6.9; PHEMS). The cells on filter fragments were washed in PHEMS and permeabilized with cold acetone $\left(-20^{\circ} \mathrm{C}\right)$ for $1 \mathrm{~min}$, followed by two washes in PHEMS. The samples were soaked in the blocking solution (Tris-buffered saline [TBS] containing 5\% ovalbumin) for $10 \mathrm{~min}$ at room temperature. Then they were incubated for 1 h $30 \mathrm{~min}$ in a moist chamber with the anti-caveolin polyclonal antibody in the blocking solution. After rinsing, the cells were incubated for $1 \mathrm{~h}$ with the secondary antibody, CY5-conjugated goat anti-rabbit IgG in the blocking solution.

For luminal uptake studies, LDL or acetylated LDL (acLDL) were prepared as described previously (Dehouck et al. 1997). The ECs were incubated for $45 \mathrm{~min}$ at $37^{\circ} \mathrm{C}$ in prewarmed Ringer HEPES LDL or acLDL $(35 \mu \mathrm{g} / \mathrm{ml})$. All incubations were terminated by three washes in Ringer HEPES $+0.2 \%$ bovine serum albumin (BSA) (cultured tested; ICN) and three washes in Ringer HEPES.
For LDL detection, cells previously incubated with LDL were fixed in ice-cold ethanol $\left(-20^{\circ} \mathrm{C}\right)$. Cells were incubated overnight at $4^{\circ} \mathrm{C}$ with polyclonal goat anti-ApoB antibodies. They were washed three times with the blocking solution and incubated $1 \mathrm{~h}$ at room temperature with the secondary antibodies. As a secondary antibody, Alexa 568 donkey anti-goat IgG (dilution 1/100 in blocking solution) was used.

Cells incubated with LDL or acLDL were washed twice with DMEM at $37^{\circ} \mathrm{C}$. Some of the cells incubated with LDL or acLDL were incubated with $100 \mu \mathrm{M}$ chloroquine for 30 and $60 \mathrm{~min}$. All samples were fixed in $4 \%$ paraformaldehyde and incubated for $1 \mathrm{~h}$ at room temperature either with antiLamp 1 (detection of endocytosed vesicles presenting lysosomal characteristics) or anti-ApoB (detection of nondegradated LDL) antibodies. Alexa 488 goat anti-rabbit or rabbit anti-goat was used at the same dilution $1 / 100$ in the blocking solution, to reveal Lamp-lor ApoB, respectively.

Following a final rinse, the filters and their attached monolayers were mounted on glass microscopic slides using Mowiol mount (Hoechst, Frankfurt, Germany). The specimens were visualized and photographed with a Leica fluorescence microscope.

The method used to quantify vesicle sizes consists in direct measurement of vesicle diameter on the screen by Adobe Photoshop measurement tools (Adobe Photoshop software, Adobe Systems, CA, USA). This method was applied to a large number of vesicles: around 1000 vesicles were counted for each condition. These measurements were obtained from three series of different incubation conditions.

For confocal microscopy, cells incubated with DiI-LDL or DiI-acLDL $(100 \mu \mathrm{g} / \mathrm{ml})$ for $45 \mathrm{~min}$ at $37^{\circ} \mathrm{C}$ were washed + $0.2 \%$ BSA (cultured tested; ICN) and three washes in Ringer HEPES. Then cells were fixed with paraformaldehyde ( $1 \%$ or $8 \%$ ) permeabilized in $0.1 \%$ Triton $\mathrm{X} 100$ for $30 \mathrm{~min}$. Then cells were incubated for $30 \mathrm{~min}$ with polyclonal goat anti-ApoB antibodies $10 \%$ in calcium- and magnesium-free PBS. Cells fixed with $1 \%$ paraformaldehyde were then incubated for $1 \mathrm{~h}$ with anti-Golgin antibody (1/100) at room temperature. Cells fixed with $8 \%$ paraformaldehyde were then incubated for $1 \mathrm{~h}$ with anti- $\beta$-COP antibody $(1 / 80)$ at room temperature. Then, cells were washed three times with calcium- and magnesium-free PBS and incubated for $1 \mathrm{~h}$ at room temperature and $1 \mathrm{~h}$ with the secondary antibodies (Alexa 488-conjugated anti-mouse IgG mouse, 1/200) in the blocking solution. Following final rinses, the filters and their attached monolayers were mounted on glass microscopic slides using Mowiol mount, the specimens were visualized and photographed with a Leica confocal microscope.

\section{Electron Microscopy}

The cells were fixed routinely with $2.5 \%$ glutaraldehyde in sodium cacodylate buffer, $\mathrm{pH} 7.2$, at $4^{\circ} \mathrm{C}$ and postfixed in $1 \%$ $\mathrm{OsO} 4$ for $60 \mathrm{~min}$. After dehydratation in ethanol and embedding in Araldite, sections were cut perpendicular to the monolayer, 
contrasted with lead hydroxide, and examined with an electron microscope (EM 420; Philips, Eindhoven, The Netherlands). The same protocol was followed after cell incubation with goldlabeled LDL made from freshly isolated LDL and 16-nm colloidal gold according to the method of Handley et al. (1981). As a control experiment, the same experiment was also performed after incubation with gold particles only without any ligand. Then cells were prepared according to the procedure described above.

\section{RESULTS}

\section{EC Criteria}

Figure $1 A$ illustrates the typical phenotype of confluent brain capillary ECs plated on an insert coated with rat tail collagen and cocultured 12 days with astrocytes. ECs form a monolayer of nonoverlapping and sprindle-shaped cells.

ECs in brain microvessels possess tight junctions of remarkably low permeability, which are to a large extent responsible for forming and maintaining the BBB in vivo (Risau and Wolburg 1990). Occludin, an integral membrane protein also associated with tight junctions, was found to be expressed in various epithelial and endothelial tissues. Immunofluorescence microscopy (Figure $1 B$ ) demonstrated that brain capillary ECs in culture clearly expressed occludin continuously at cell-cell contacts, indicating that these cells are circumscribed by continuous junctional structures, as would be expected for a tight paracellular barrier. More recently, the claudins have been proven to be one of the essential proteins for tight junction strands and the composition of the claudin species directly determines the barrier function (Furuse et al. 1998 1999; Wolburg and Lippoldt 2002; Ishizaki et al. 2003). More precisely, claudin- 5 has been described to play a central role in the endothelial tight junction barrier regulation (Nitta et al. 2003; Ohtsuki et al. 2007). Our immunofluorescent staining revealed a strong linear pattern of staining with a perijunctional organization (Figure $1 C$ ). These findings are consistent with our earlier results concerning the high electrical resistance ( 500 to $800 \Omega \cdot \mathrm{cm}^{2}$ ) and the low permeability of our bovine brain capillary EC monolayer (Cecchelli et al. 1999).

To determine whether caveolae could be present in these ECs, we performed indirect immunofluorescent analyses on ethanol fixed ECs cocultured with astrocytes for 12 days using commercially available affinity-purified polyclonal antibodies against caveolin. Caveolin, a 21 - to $24-\mathrm{kDa}$ integral membrane protein, is a major structural component of caveolae membranes in vivo (Rothberg et al. 1992; Kurzchalia et al. 1992). Brain capillary ECs exhibited intense staining: an heterogeneous punctuate staining over the body of the cells (Figure 2A) and a distinct bright linear staining along portions of the cell borders (Figure 2B).

To determine whether the structures identified by indirect immunofluorescence corresponded to cell surface invaginations and/or to populations of subplasmalemmal vesicles with the
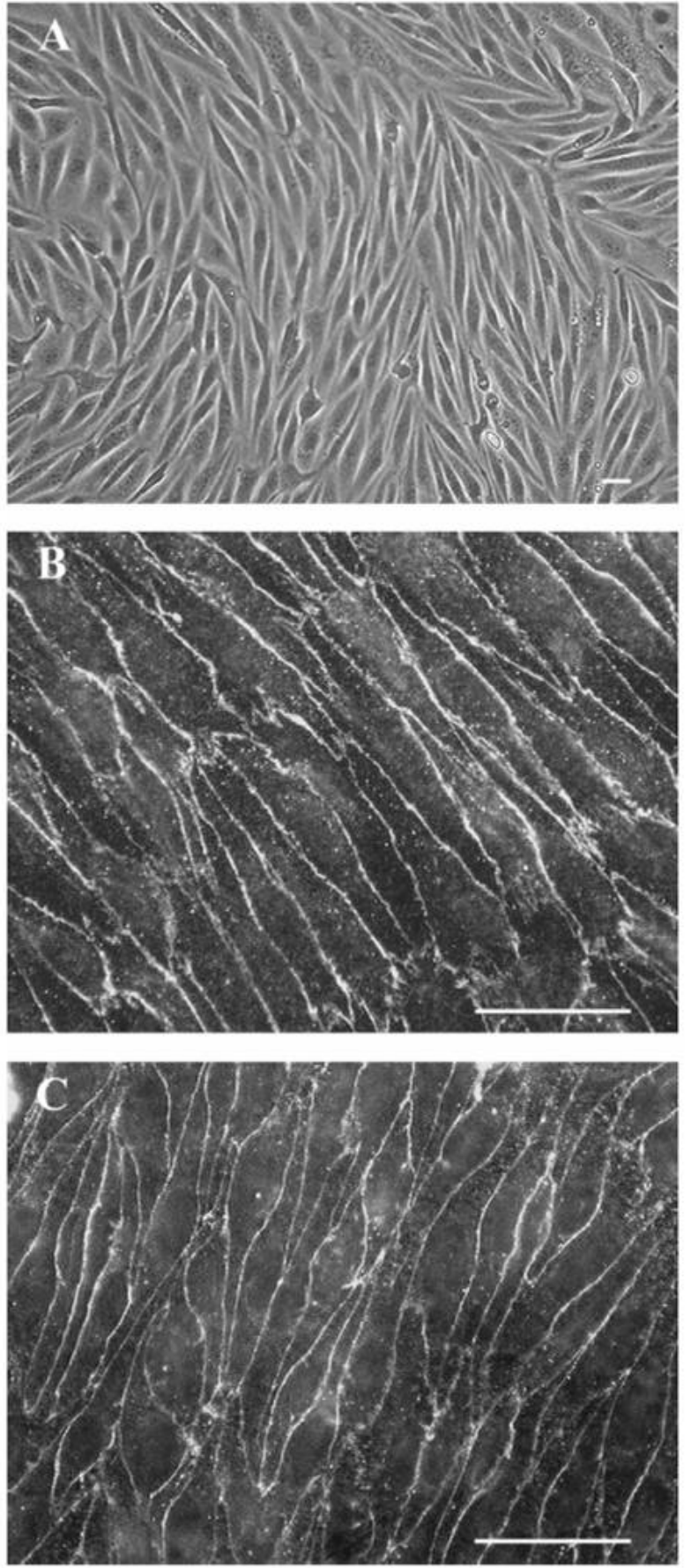

FIG. 1. Phase-contrast micrograph of confluent brain capillary endothelial cells grown on the upper face of a collagen coated filter. Occludin $(B)$ and claudin-5 $(C)$ immunofluorescent labeling of ECs. The cortical staining reveals that these ECs are sealed by tight junctions. Bars $=25 \mu \mathrm{m}$.

morphological features characteristic of caveolae, EC monolayers were processed for examination by thin-section electron microscopy. Flask-shaped invaginations of the plasma membrane, about $60 \mathrm{~nm}$ in diameter, were detected in all cells examined. Typically 10 or more caveolae were grouped in clusters, which were observed along both plasmalemmal 

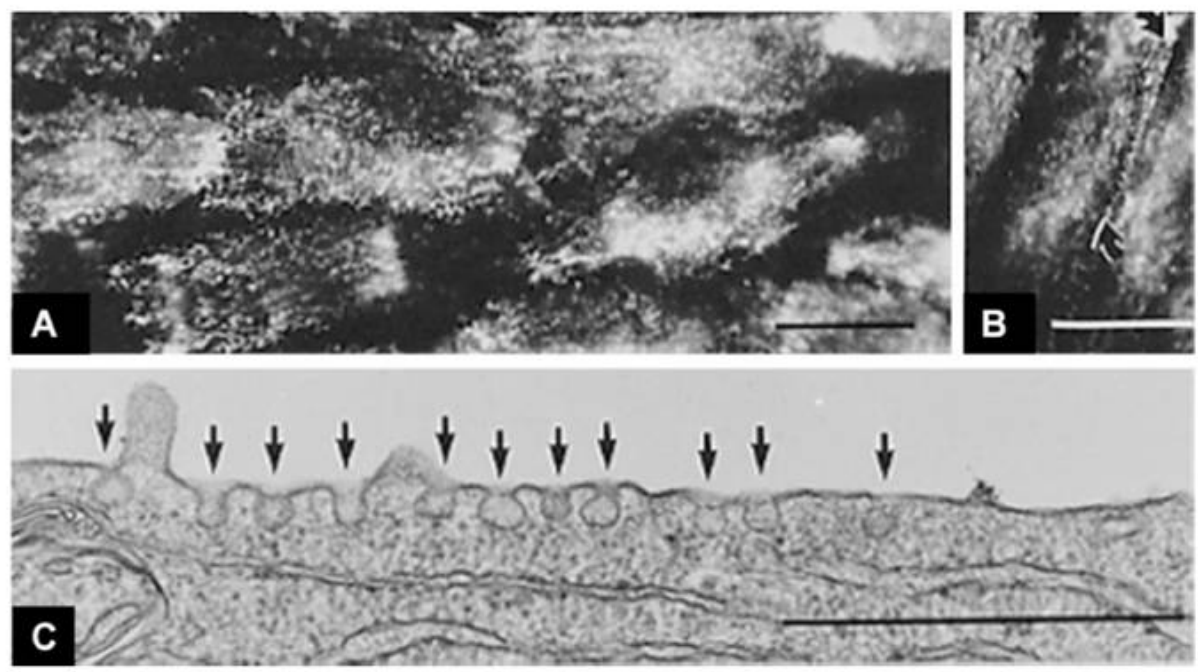

FIG. 2. Indirect immunofluorescent staining on ethanol-fixed ECs cocultured with astrocytes during 12 days using a commercially available affinity-purified polyclonal antibodies against caveolin $(A)$. In those cells that exhibited intense staining, there was a distinct bright linear staining along portions of the cell borders $(B$, curved arrows). Electron micrograph shows typical membrane invaginations found at the brain capillary EC surface $(C$, arrows). Numerous budding profiles lacking clathrin coats and resembling caveolae were found at the plasma membrane. In $A$ and $B$, bars $=25 \mu \mathrm{m}$. In $C$, bar $=1 \mu \mathrm{m}$.

surfaces. Individual caveolae appeared to be in continuity with the plasmalemmal surface, and thus opened to the extracellular space (Figure $2 C$ ). Single caveolae were rarely detected. During the electron microscopy study described above, we detected few, if any, clathrin-coated invaginations on the EC surfaces.

Taken together, these data indicate that ECs cocultured with astrocytes develop a morphology that closely resembles the endothelium in situ. These highly differentiated ECs, mimicking the in vivo situation, provide a model to study the mechanism of transcellular transport that exists at the BBB level.

\section{Colocalization of the LDL Receptor and Caveolin by Cell Fractionation Experiments}

Preliminary studies have suggested that caveolae might be involved in the transport of LDL from the blood to the brain (Dehouck et al. 1997). To determine whether the LDL receptor is really located in caveolar membranes, we first performed subcellular fractionation experiments.

Using the approach described by Smart et al. (1995), we isolated several distinct membrane compartments and used immunoblotting to determine whether EC caveolin-containing fractions isolated in the absence of nonionic detergent contain the LDL receptor (Figure 3). Although the LDL-receptor is present in the total plasma membranes (lane 1), its relative abundance in the caveolin fraction (lane 2) was not detectable under the loading and detection conditions used here.

However, we have already demonstrated that the lipid requirement of astrocytes increases the expression of EC LDL receptors (Dehouck et al. 1994). A sixfold increase in binding of LDL to ECs cultured with cholesterol-depleted astrocytes was obtained. The fractionation experiment described above was performed using these "up-regulated" cells. As shown in Figure 3, lane 3, using the same protocol, a 135-kDa protein corresponding to the LDL receptor was detectable in the caveolae fraction. Clathrin heavy chain has never been detected in the caveolae fraction, although it was abundant in the starting homogenate, indicating that our fraction did not contain coated-pit derived membrane fractions (not shown).

Paxillin (Figure 3) and FAK (data not shown) were analyzed by Western blots in the fraction collected from the resulting Optiprep gradients. Paxillin and FAK, which are negative markers of caveolae domains in ECs (Demeule et al. 2000), were only detected in the total plasma membrane fractions (lane 1) but not in the caveolae fraction (lanes 2 and 3 ). As caveolae have already been described as the clustering site of many different

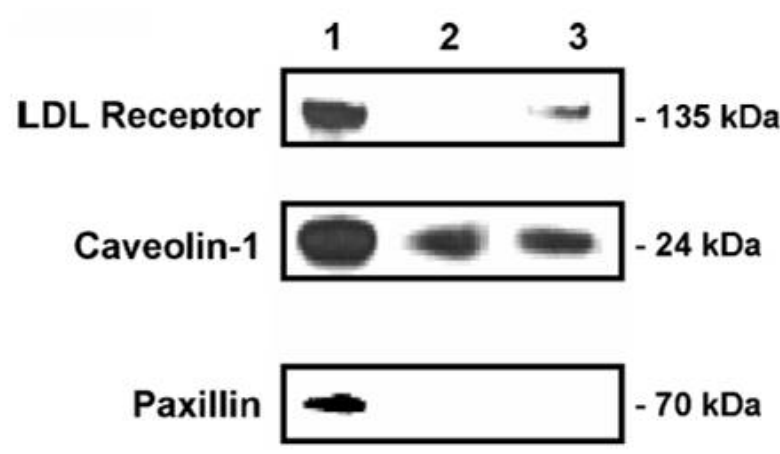

FIG. 3. Immunoblot detection of proteins enriched in caveolae. Two fractions from ECs in coculture ( 12 ) or from up-regulated cells (3) were used in the standard immunoblot assay; $10 \mu \mathrm{g}$ of whole cell lysate (1), $5 \mu \mathrm{g}$ of caveolae $(2,3)$. Each of these samples was separated on $4 \%$ to $15 \%$ SDS/polyacrylamide gel and immunoblotted with anti-LDL receptor, anti-caveolin, or anti-paxillin antibodies as described in Materials and Methods. 

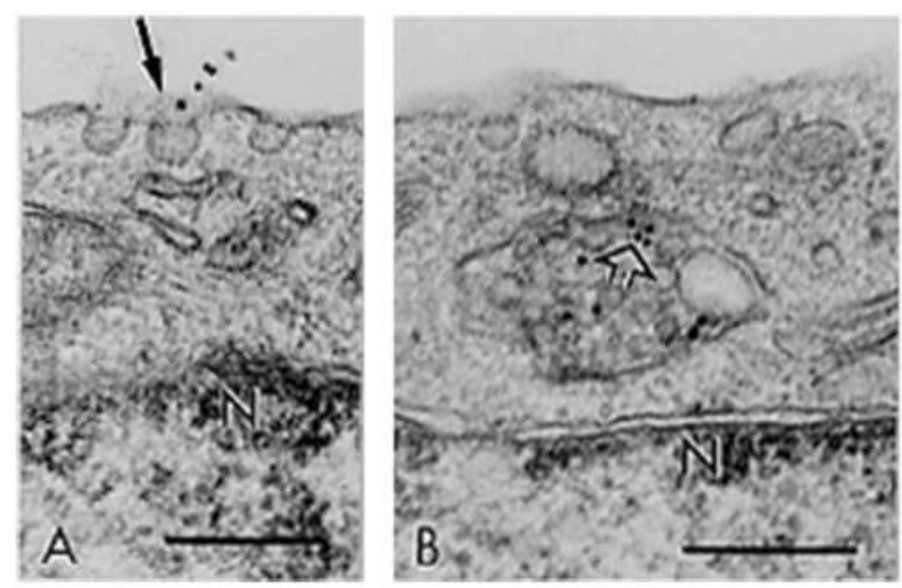

FIG. 4. Description of the endosomal "clathrin-independent" LDL endocytotic pathway in ECs. At early time of incubation LDL-Au are observed near plasmalemmal vesicles, caveolae $(A$, arrow). Internalized luminally administered gold-labeled LDLs were detected, after 45 min of incubation, in multivesicular structure $(B)$ and appear to be segregated outside internal profiles (open arrow). $\mathrm{N}=$ nucleus; bar $=0.25 \mu \mathrm{m}$.

receptors capable of being internalized in the cell, we went on to determine whether these LDL receptor-rich caveolae structures are involved in LDL transcytosis within brain capillary ECs.

\section{Caveola Mediated-Endocytosis}

The caveolae-mediated endocytosis in brain capillary ECs was explored by electron microscopy. We have already demonstrated that during transcytosis, LDL reaches the abluminal face of the ECs without any degradation, in contrast to acLDLs, which were totally degraded by these cells after endocytosis (Dehouck et al. 1997). To track the pathway of the conventional LDL-receptor ligand, colloidal gold-conjugated LDL (LDL-Au) uptake was investigated to define the primary compartments involved in receptor-mediated endocytosis. At early time of incubation, LDL-Au complexes were observed near caveolae structures (Figure $4 A$ ). After a 45 -min cell incubation with LDLAu complexes, gold particles were detected in typical spherical structures containing numerous empty vesicular inclusions (Figure $4 B$ ). In these vesicles, LDL-Au segregated outside of the internal profiles as if caveolae were fused with preexisting or induced endosomal compartments and turned inside out with a release of the caveolar content. No gold particle was observed in endothelial cells when the same experiment was performed with Au only (data not shown).

In all sections studied, clathrin-coated vesicles were rarely observed and never contained LDL-Au complexes.

All these observations suggested that these multivesicular bodies of endosomal structure result from a caveolae accumulation and may be related to caveosomes described by Pelkmans et al. (2001), as it seems to be characteristic of caveolar uptake pathway.

\section{Characterization of the Compartment Involved in LDL Transcytosis}

This caveosome is depicted as a pathway for endocytosis. This organelle is distinct from endosome and secretory vesicles by its internal $\mathrm{pH}$, which is estimated to be near neutral. To get further insights about the $\mathrm{pH}$ of the compartment involved in LDL transport through our blood-brain capillary EC monolayer, we used an acidotropic amine reagent, such as chloroquine, to characterize the late endosome-containing native LDL.

After a 45-min incubation of brain capillary ECs with LDL or acLDL, vesicles were revealed using anti-Lamp-1 and ligands using anti-ApoB antibodies. AcLDL were used here as a marker of the degradative EC pathway (Dehouck et al. 1997). We therefore investigated the effect of chloroquine treatment on monolayers loaded with LDL or acLDL and compared the shifts in vesicle diameters. Before chloroquine treatment, all Lamp-1-positive vesicles had the same mean diameter, from 400 to $500 \mathrm{~nm}$, for both LDL- and acLDL-loaded cells (Figure $5 A$ and $B$, bars). Then, in acLDL-incubated cells, the osmotic

A

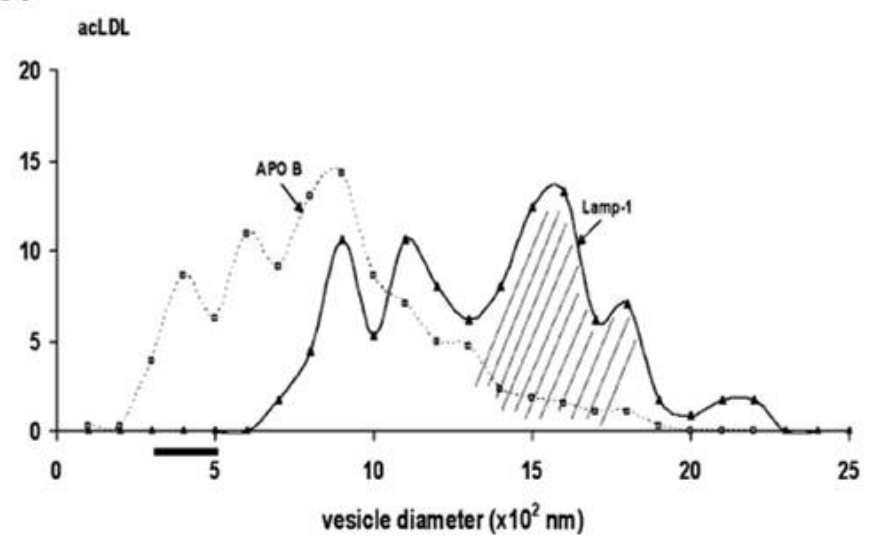

B

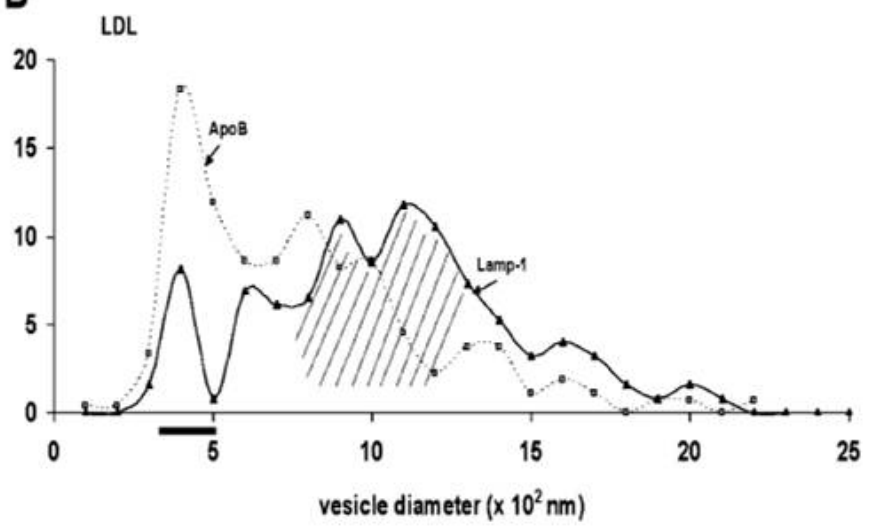

FIG. 5. Vesicle diameter measurements in acLDL- $(A)$ or LDL- $(B)$ incubated cells were performed on lipoprotein-loaded ECs, treated (curves) or not (bars) with chloroquine $(100 \mu \mathrm{M})$ for $60 \mathrm{~min}$ at $37^{\circ} \mathrm{C}$. Intracellular compartments were revealed by anti-Lamp 1 antibodies (solid lines) and ligands were followed by anti-apoB antibodies (dashed lines). 

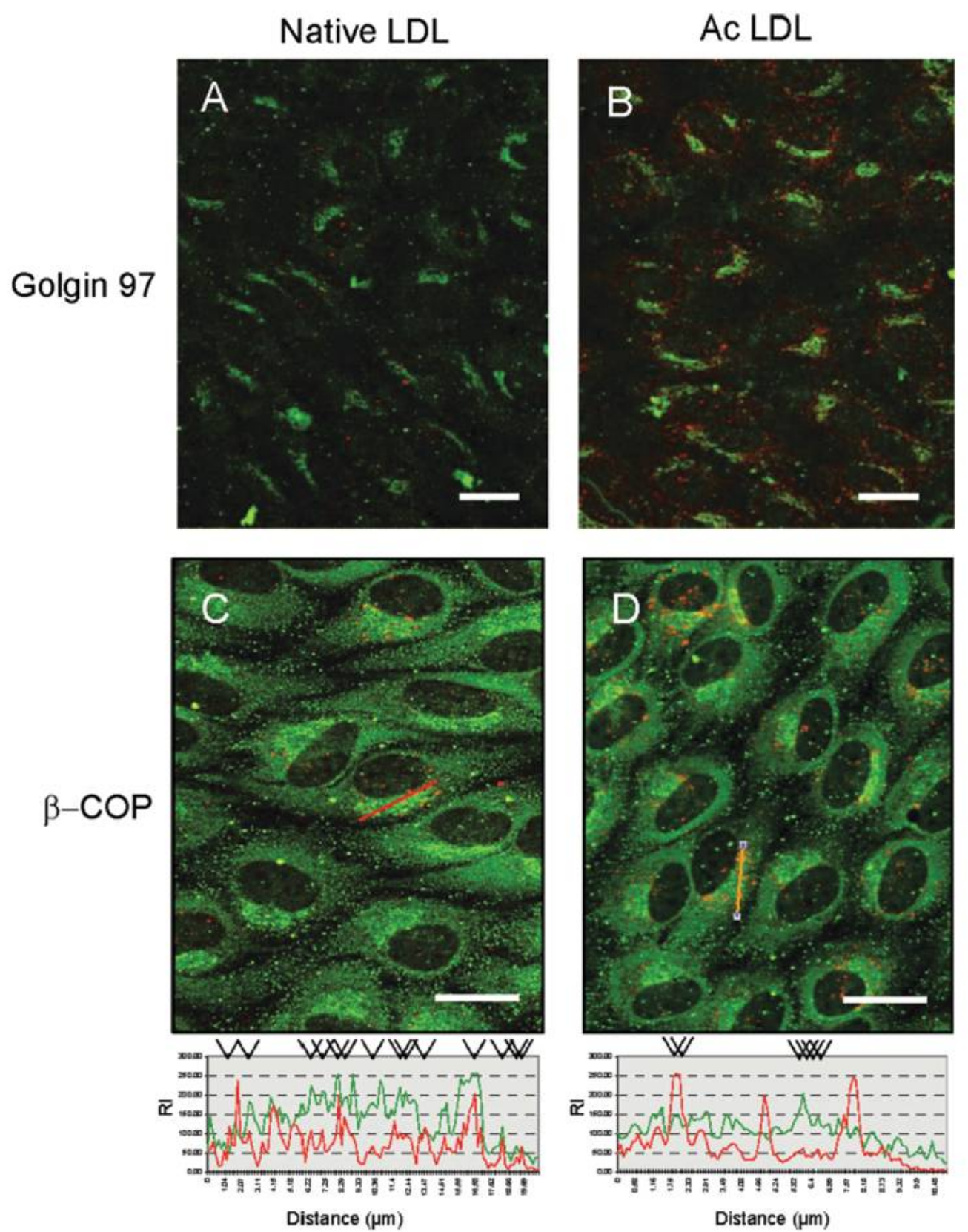

FIG. 6. Subcellular localisation of native or acLDL in brain capillary ECs. ECs incubated with DiI native (A, C: red) or Dil acLDL $(B, D:$ red) were fixed and labeled with anti Golgin $97(A, B)$ or anti $\beta$-COP $(C, D)$ followed by Alexa fluor 488 secondary antibodies (green). Although no colocalization was demonstrated between Golgin 97 and lipoproteins $(A, B)$, confocal fluorescence microscopy shows some colocalization between native LDL and $\beta$-COP (graphs under micrographs). Arrowheads indicate colocalizing structures largely more observed in native LDL-incubated ECs. Bars $=20 \mu \mathrm{m}$.

shock induced by the chloroquine treatment caused a huge increase in the vesicle sizes (Figure $5 A$, dashed area under the curve) compared to untreated cells (Figure $5 \mathrm{~A}$, bar). The distribution of vesicle sizes shifted drastically, from 500 to 1500 to $2000 \mathrm{~nm}$, indicating that we are dealing with an acidic Lamp-1positive compartment. Interestingly, these acidic vesicles were not revealed by anti-ApoB antibodies (Figure $5 \mathrm{~A}$, dashed area).

In contrast, in chloroquine-treated LDL-loaded cells, the size shift was less pronounced (Figure 5B, bar compared to dashed area under the curve). The labeling mostly concerned vesicles with a mean diameter from 500 to $1500 \mathrm{~nm}$, which can be considered as mildly acidic vesicles devoid of lipoprotein degradation activity, as demonstrated by the presence of anti-ApoB labeling in the dashed region (Figure $5 B$ ).

To further characterize the intracellular compartment in which LDL and acLDL reside, two-color colocalization studies were carried out between DiI LDL and $\beta$-COP, a caveosome marker (Norkin et al. 2002), or golgin 97, a specific Golgi marker 


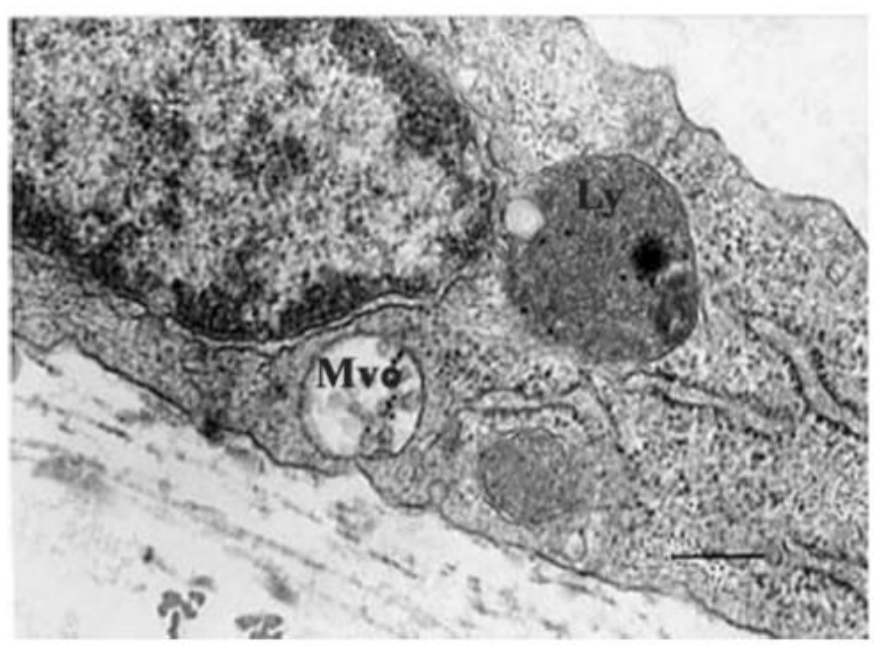

FIG. 7. Electron microscopy micrograph of brain capillary ECs depicts a different morphology between a multivesicular organelle (Mvo) and a lysosomal vesicle (Ly). Bar $=0.25 \mu \mathrm{m}$.

(Griffith et al. 1997). Under the conditions of these experiments, there was no cross-over between Alexa Fluor 48 (green) and DiI (red) emission. The morphology of the Golgi apparatus, as determined by staining with golgin 97 , did not correspond with DiI-LDL (Figure 6A) nor DiI-acLDL (Figure 6B), suggesting that the majority of lipoproteins does not reside within this compartment. In contrast, intracellular DiI-LDL showed some colocalization with $\beta$-COP (Figure $6 C$ ). This colocalization was not observed for DiI ac-LDL (Figure $6 D$ ). Interestingly, acLDL being degraded by the ECs presented a clear perinuclear localization, consistent with a lysosomal accumulation (Figure 6B).

The existence of two different endosomal compartments involved in LDL or acLDL trafficking is also confirmed by the morphological differences clearly seen by electron microscopy (Figure 7). Interestingly, the multivesicular organelle involved in LDL transcytosis was located near the abluminal face of ECs.

\section{DISCUSSION}

\section{In Vitro BBB}

The structural basis of the BBB is dependent on the properties of specialized brain capillary ECs. Largely based on the vicinity of vascular brain ECs to astrocytes, it was proposed that the neural environment might be responsible for the development of the BBB properties. By growing primary ECs on one side of a porous filter and astrocytes on the other, we are able to reconstruct the unique environment that exists in vivo and induce in vitro most of the BBB characteristics (Cecchelli et al. 1999, 2007). Among these characteristics, one structural element is of major importance for ensuring and maintaining brain homeostasis. Brain capillary ECs are coupled by tight junctions of extremely low permeability, thus preventing paracellular flux of solutes. From the perspective of the tightness of this barrier, brain capillary ECs differ considerably from others in phenotype. In accordance with results of Hirase et al. (1997) and Ohtsuki et al. (2007), high levels of occludin and claudin-5 expression were found in our brain capillary ECs, demonstrating that they present a highly differentiated phenotype.

This observation is reinforced by our immunofluorescent results showing extensive staining of caveolin in brain capillary ECs and relative abundance of caveolae in the cells. Indeed, elevated expression of caveolin proteins and these organelles are generally associated with cell differentiation (Galbiati et al. 1998; Régina et al. 2004).

These data, added to previous ones (Dehouck et al. 1992; Lundquist et al. 2002), demonstrate that this model closely mimics the in vivo situation, and allows studies at the cellular level to be carried out. Here, we have used this model to determine the intracellular compartments involved in blood-borne macromolecule transcytosis across the BBB.

\section{Caveolae as the First Step of LDL Transcytotic Pathway through the Brain Capillary Endothelial Cells}

In contrast to peripheral vessels, in which LDL receptor is down-regulated, confluent brain capillary ECs express an LDL receptor in spite of their contact with physiological concentrations of lipoprotein (Méresse et al. 1989). As demonstrated using our in vitro model (Méresse et al. 1989; Dehouck et al. 1994), this luminal receptor is likely to be a bona fide LDL receptor, as indicated by its saturability, its specificity, and its molecular weight of $135 \mathrm{kDa}$. The discovery of this LDL receptor on the luminal side of the BBB of ECs, together with the finding that enzymes involved in the lipid metabolism are present within the brain (Albers et al. 1992), led us to suggest that LDL might cross the BBB, although most aspects of this pathway are still unclear.

Generally, in endothelia, uptake of macromolecules is known to follow a dual pathway, receptor-mediated endocytosis and transcytosis. In fact, this is the case for numerous plasma proteins that are taken up and their metabolites are used by ECs or are transported to the adjacent tissues and cells. Data demonstrated that in vivo, caveolae are present on both sides of the ECs, indicating that these vesicles are involved in a transcellular transport process (Virgintino et al. 2002). However, for a given molecule, the endocytosis/transcytosis ratio may vary with the physiological state of the vascular bed involved (for review see Simionescu and Simionescu 1991). In our highly differentiated brain, LDL does not follow the classical degradation pathway but instead undergoes transcytosis through the ECs by a receptor-mediated process, bypassing the lysosomal compartment (Dehouck et al. 1997). Moreover, preliminary studies have shown that filipin treatment, a sterol-binding agent causing the disassembly of caveolae (Rothberg et al. 1990), completely abolished LDL endocytosis, suggesting that caveolae might be involved in the LDL transport from the blood to the brain. In this paper, we clearly demonstrate, by using the detergent-free method described by Smart et al. (1995) and 
biochemical approach, that the LDL receptor expressed at the brain capillary EC luminal face is located in caveolae.

In basal conditions, we never succeeded in detecting the LDL receptor in the caveolae rich fraction. However, the vascular system is lined with a monolayer of highly differentiated ECs that are strategically located to form the critical barrier that regulates exchanges between circulating blood and the underlying tissue cells. ECs are quite sensitive to the local environment and may modulate their phenotype and barrier function according to the needs of the underlying tissue cells. For example, we demonstrated that the lipid requirement of astrocytes increases the number of LDL receptor at the luminal side of brain capillary ECs (Dehouck et al. 1994). Our present biochemical assays are in total agreement with these previous results, showing that for equal amount of caveolae protein fractions loaded onto the gel, we detected the LDL receptor only in caveolae fraction from upregulated cells. Interestingly, the increase in the number of LDL receptors at the cell surface in up-regulated cells was followed by an increase in LDL endocytosis and transcytosis (Dehouck et al. 1997). These results are in accordance with those of Stewart (2000) who demonstrates that enhanced BBB penetration of some molecules may be due to regulated expression of specific receptors, rather than activation of the transcytosis mechanism in brain capillaries.

\section{Multivesicular Organelle Characterization}

We have previously demonstrated that brain capillary ECs express all the enzymes required for lipoprotein degradation, acLDL being degraded by these cells (Dehouck et al. 1997). AcLDL-incubated cells were thus used as a control for lysosomal compartment formation in brain capillary ECs. When cells are loaded with LDL or acLDL, fluorescent immunolabeling experiments reveal that the two intracellular compartments present the same size. These compartments in which ApoB epitope was still detectable can be considered as the first steps of acLDL intracellular traffic. Nevertheless, a 45-min incubation time was sufficient to deliver our tracers to endosome that had time to acidify and acquire lysosomal enzymes. Using chloroquine, a weak base that accumulates in acidic compartments, we detected such acidic vesicles only in acLDL-incubated cells. In these Lamp1-positive vesicles, it was impossible, using anti-ApoB antibodies, to detect ApoB epitope, indicating that the apolipoprotein is degraded in the lysosomal compartment. These results are consistent with the degradation capacities of brain capillary ECs.

In contrast, after chloroquine treatment, in LDL-incubated cells where LDL were not degraded, only mildly acidic ApoBpositive vesicles were detected. These observations are in accordance with our previous results demonstrating that LDL are transcytosed through the brain endothelial cells and found intact in the abluminal compartment (Dehouck et al. 1994). From the point of view of $\mathrm{pH}$, cellular location, and caveolaederived formation, multivesicular structures described here can be related to the caveosome structure involved in the trans- port of the uncoated virus simian virus 40 (SV40) and cholera toxin as reviewed in Parton and Richards (2003). Even if little is known about caveosome, Norkin and Kurskin (2005) have clearly demonstrated that this endosomal compartment was marked by $\beta$-COP, like the Golgi, but did not contain the Golgi marker golgin 97 (a peripheral membrane protein that appears to be localized exclusively on the cytoplasmic face of the Golgi [Griffith et al.1997]), implying that they are distinct from the Golgi. Our results confirm that, at the BBB level, we are dealing with a same compartment involved in a transcytotic process allowing the LDL delivery from the blood to the brain parenchyma.

Then, in accordance with Spencer and Verma (2007), confirming the use of the LDL receptor for specific brain drug delivery, this work presents, for the first time, a detailed endocytotic process for LDL transcytosis through the BBB. Furthermore, our results clearly argue in favor of a cholesterol transport from the circulation to the brain. Even if this pathway can be considered as of little importance under normal conditions, the recent data demonstrating that the lack of LDL receptor aggravates learning deficits and amyloid deposits in Alzheimer transgenic mice has to lead us to reconsider this position (Cao et al. 2006). Indeed, the role of the LDL receptor at the brain capillary level is still reinforced by recent observations in studies demonstrating that statins may have a protective effect on the development of dementia (Arvanitakis et al. 2008). According to the data reported thus far, there is no difference between the protective effect of hydrophobic and hydrophilic statins. If statin effects on the brain can be confirmed, then they are likely to be of indirect nature and mediated by the microcirculation (Kirsch et al. 2003). The determination of the role of LDL receptor in the development of Alzhemer disease is of particular significance because the receptor is the secondary target for statin treatment (Brown and Goldstein 1986). Further works are now in progress to study the implication of this transcellular pathway in pathological conditions in a view to develop new therapeutic strategies for the treatment of neurodegenerative diseases.

\section{REFERENCES}

Albers J.J., Tollefson J.H., Wolfbauer G., and Albright R.E. Jr. (1992) Cholesteryl ester transfer protein in human brain. Intrenational Journal of Clinical Laboratory Research, 21, 264-266.

Arvanitakis Z., Schneider J.A., Wilson R.S., Bienias J.L., Kelly J.F., Evans D.A., and Bennett D.A. (2008) Statins, incident Alzheimer disease, change in cognitive function, and neuropathology. Neurology, 70, 1795-1802.

Basu S.K., Goldstein J.L., Anderson G.W., and Brown M.S. (1976) Degradation of cationized low density lipoprotein and regulation of cholesterol metabolism in homozygous familial hypercholesterolemia fibroblasts. Proceedings of the National Academy of Sciences of the United States of America, 73, 31783182.

Betz A.L., and Goldstein G.W. (1978) Polarity of the blood-brain barrier: Neutral amino acid transport into isolated brain capillaries. Science, 202, 225227.

Booher J., and Sensenbrenner M. (1972) Growth and cultivation of dissociated neurons and glial cells from embryonic chick, rat and human brain in flask cultures. Neurobiology, 2, 97-105. 
Bornstein M.B. (1958) Reconstituted rat tail collagen used as substrate for time tissue cultures on coverslips in Maximow slides abd roller tubes. Laboratory Investigation, 7, 134-139.

Brown M.S., and Goldstein J.L. (1986) A receptor-mediated pathway for cholesterol homeostasis. Science, 232, 34-47.

Cao D., Fukuchi K., Wan H., Kim H., and Li L. (2005) Lack of LDL receptor aggravates learning deficits and amyloid deposits in Alzheimer transgenic mice. Neurobiology of Aging, 27, 1632-1643.

Cecchelli R., Berezowski V., Lundquist S., Culot M., Renftel M., Dehouck M.P. and Fenart L. (2007) Modelling of the blood-brain barrier in drug discovery and development. Naturre Review in Drug Discovery, 6, 650-661.

Cecchelli R., Dehouck B., Descamps L., Fenart L., Buée-Scherrer V., Lundquist S., Rentfel M., Torpier G., and Cecchelli R. (1999) In vitro model for evaluating drug transport across the blood-brain barrier. Advanced Drug Deliven Reviews, 36, 165-178.

Dehouck B., Dehouck M.P., Fruchart J.C., and Cecchelli R. (1994) Upregulation of the low density lipoprotein receptor at the Blood-brain barrier: Intercommunication between brain capillary ECs and astrocytes. Journal of Cell Biology, 126, 465-473.

Dehouck B., Fenart L., Dehouck M.P., Pierce A., Torpier G., and Cecchelli R. (1997) A new function of the LDL receptor: Transcytosis of LDL across the Blood-brain barrier. Journal of Cell Biology 138, 877-889.

Dehouck M.P., Jolliet-Riant P., Brée F., Fruchart J.C., Cecchelli R., and Tillement J.P. (1992) Drug transfer across the blood-brain barrier: Correlation between in vitro and in vivo models. Journal of Neurochemistry, 58, 1790 1797.

Demeule M., Jodoin J., Gringras D., and Beliveau R. (2000) P-glycoprotein is localized in caveolae in resistant cells and in brain capillaries. FEBS Letters, 466, 219-224.

Furuse M., Fujita K., Hiiragi T., Fujimoto K., and Tsukita S. (1998) Claudin1 and -2 : Novel membrane proteins localizing at tight junctions with no sequence similarity to occludin. Journal of Cell Biology 141, 1539-1550.

Furuse M., Sasaki H., and Tsukita S. (1999) Manner of interaction of heterogeneous claudin species within and between tight junction strands. Journal of Cell Biology, 147, 891-903.

Galbiati F., Volonte D., Engelman J.A., Watanabe G., Burk R., Pestell R.G., and Lisanti M.P. (1998) Targeted downregulation of caveolin-1 is sufficient to drive cell transformation and hyperactivate the p42/44 MAP kinase. EMBO Journal, 16, 6633-6648.

Griffith K.J., Chan E.K., Lung C.C., Hamel J.C., Guo X., Miyachi K., and Fritzler M.J. (1997) Molecular cloning of a novel 97-kd Golgi complex autoantigen associated with Sjögren's syndrome. Arthritis and Rheumatism, 40, 1693-1702.

Handley D.A., Arbeeny C.M., and Eder H.A. (1981) Hepatic binding and internalization of low density lipoprotein-gold conjugates in rats treated with 17 a-ethinyl estradiol. Journal of Cell Biology,90, 778-785.

Hirase T., Staddon J.M., Saitou M., Ando-Akatsuka Y., Itoh M., Furuse M., Fujimoto K., Tsukita S., and Rubin L.L. (1997) Occludin as a possible determinant of tight junction permeability in ECs. Journal of Cell Science, 110. $1603-1613$.

Howland D.S., Trusko S.P., Savage M.J., Reaume A.G., Lang D.M., Hirsch J.D. Maeda N., Siman R., Greenberg B.D., Scott R.W., and Flood D.G. (1998) Modulation of secreted $\beta$-amyloid precursor protein and amyloid $\beta$-peptide in brain by cholesterol. Journal of Biological Chemistry, 273, 16576-16582.

Ishizaki T., Chiba H., Kojima T., Fujibe M., Soma T., Miyajima H., Nagasawa K., Wada I., and Sawada N. (2003) Cyclic AMP induces phosphorylation of claudin- 5 immunoprecipitates and expression of claudin- 5 gene in blood-brain-barrier endothelial cells via protein kinase A-dependent and -independent pathways. Experimental Cell Research, 290, 275-288.

Kenagy R., Bierman E.L., Schwartz S., and Albers J.J. (1984) Metabolism of low density lipoprotein by bovine ECs as a function of cell density-Arteriosclerosis, 4, 365-371.

Kirsch C., Eckert G.P., Koudinov A.R., and Müller W.E. (2003) Brain cholesterol, statins and Alzheimer's Disease. Pharmacopsychiatry, 36, 113-119.
Kurzchalia T.V., Dupree P., Parton R.G., Kellner R., Virat H., Lenhert M., and Simons K. (1992) VIP21, a 21-kD membrane protein is an integral component of trans-Golgi-network-derived transport vesicles. Journal of Cell Biology, 118, 1003-1014.

Lucarelli M., Borrelli V., Fiori A., Cucina A., Granata F., Potenza R.L., Scarpa S., Cavallaro A., and Strom R. (2002) The expression of native and oxidized LDL receptors in brain microvessels is specifically enhanced by astrocytesderived soluble factor(s). FEBS Letters, 522, 19-23.

Lundquist S., Renftel M., Brillault J., Fenart L., Cecchelli R., and Dehouck M.P. (2002) Prediction of drug transport through the blood-brain barrier in vivo: A comparison between two in vitro models. Pharmacy Research 19, 976-981.

Malavolti M., Fromm H., Ceryak S., and Shenan K.L. (1991) Cerebral lowdensity lipoprotein (LDL) uptake is stimulated by acute bile drainage. Biochimica Biophysica Acta, 108, 106-108.

Méresse S., Dehouck M.P., Delorme P., Fruchart J.C., and Cecchelli R. (1991) Lipoproteins and reconstituted blood-brain barrier.In Pharmaceutical Applications of Cell and Tissue Culture to Drug Transport, pp. 217229.

Méresse S., Delbart C., Fruchart J.C., and Cecchelli R. (1989) Low-density lipoprotein receptor on endothelium of brain capillaries.Journal of Neurochemistry, 53, 340-345.

Morris C.M., Keith A.B., Edwardson J.A., and Pullen R.G.L. (1992) Uptake and distribution of iron and transferrin in the adult rat brain. Journal of Neurochemistry, 59, 300-306.

Nitta T., Hata M., Gotoh S., Seo Y., Sasaki H., Hashimoto N., Furuse M., and Tsukita S. (2003) Size-selective loosening of the blood-brain barrier in claudin-5-deficient mice. Journal of Cell Biology, 161, 653-660.

Norkin L.C., Anderson H.A., Wolfrom S.A., and Oppenheim A. (2002) Caveolar endocytosis of simian virus 40 is followed by brefeldin A-sensitive transport to the endoplasmic reticulum, where the virus disassembles. Journal of $V_{i}$ rology, 76, 5156-5166.

Norkin L.C., and Kuksin D. (2005) The caveolae-mediated sv40 entry pathway bypasses the golgi complex en route to the endoplasmic reticulum.Virological Journal, 2, 38-44.

Ohtsuki S., Sato S., Yamaguchi H., Kamoi M., Asashima T., and Terasaki T. (2007) Exogenous expression of claudin-5 induces barrier properties in cultured rat brain capillary endothelial cells. Journal of Cellular Physiology, 210, $81-86$.

Parton R.G., and Richards A.A. (2003) Lipid rafts and caveolae as portals for endocytosis: New insight and common mechanisms. Traffic, 4, 724-738.

Pelkmans L., Burli T., Zerial M., Helenius A. (2004) Caveolin-stabilized membrane domains as multifunctional transport and sorting devices in endocytic membrane traffic. Cell, 118,:767-780.

Pelkmans L., Kartenbeck J., and Helenius A. (2001) Caveolar endocytosis of simian virus 40 reveals a new two-step vesicular-transport pathway to the ER. Nature Cell Biology,3, 473-483.

Régina A., Jodoin J., Khoueir P., Rolland Y., Berthelet F., Moumdjian R., Fenart L., Cecchelli R., Demeule M., and Béliveau R. (2004) Down-regulation of caveolin-1 in glioma vasculature: Modulation by radiotherapy. Journal of Neuroscience Research, 75, 291-299.

Refolo L.M., Malester B., LaFrancois J., Bryant-Thomas T., Wang R., Tint G.S., Sambamurti K., Duff K., and Pappolla M.A. (2000) Hypercholesterolemia accelerates the Alzheimer's amyloid pathology in a transgenic mouse model. Neurobiological Disease, 7, 321-331.

Rothberg K.G., Henser J.E., Donzell W.C., Ying Y.S., Glenney J.R., and Anderson R.G.W. (1992) Caveolin, a protein component of caveolae membrane coats. Cell, 68, 673-682.

Rothberg K.G., Ying Y.S., Kamen B.A., and Anderson R.G. (1990) Cholesterol controls the clustering of the glycophospholipid-anchored membrane receptor for 5-methyltetrahydrofolate. Journal of Cell Biology, 111, 29312938.

Shie F.S., Jin L.W., Cook D.G., Leverenz J.B., and LeBoeuf R.C. (2002) Dietinduced hypercholesterolemia enhances brain A beta accumulation in transgenic mice. Neuroreport, 13, 455-459. 
Simionescu M., and Simionescu N. (1991) Endothelial transport of macromolecules: Transcytosis and endocytosis. A look from cell biology. Cell Biology Review, 25, 5-78.

Smart E.J., Ying Y.S., Mineo C., and Anderson R.G. (1995) A detergent-free method for purifying caveolae membrane from tissue culture cells. Proceed ings of the National Academy of Sciences of the United States of America, 92, 10104-10108.

Stewart P.A. (2000) Endothelial vesicles in the blood-brain barrier: Are they related to permeability? Cellular and Molecular Neurobiology, 20, 149-163.

Spencer B.J., and Verma I.M. (2007) Targeted delivery of proteins across the blood-brain barrier. Proceedings of the National Academy of Sciences of the United States of America, 104, 7315-7316.
Traber M.G., and Kayden HJ. (1984) Vitamin E is delivered to cells via the high affinity receptor for low-density lipoprotein. American Journal of Clinical Nutrition, 40, 747-751.

Vasile E., Simionescu M., and Simionescu N. (1983) Visualization of the binding, endocytosis, and transcytosis of low density lipoprotein in the arterial endothelium. in situ. Journal of Cell Biology, 96, 1677-1689.

Virgintino D., Robertson D., Errede M., Benagiano V., Girolamo F., Maiorano E., Roncali L., and Bertossi M. (2002) Expression of Caveolin-1 in human brain microvessels. Neuroscience, 115, 145-152.

Wolburg H., and Lippoldt A. (2002) Tight junctions of the blood-brain barrier: Development, composition and regulation. Vascular Pharmacology, 38, 323337. 
